\title{
Should a robot guide like a human? A qualitative four-phase study of a shopping mall robot
}

\author{
Päivi Heikkilä ${ }^{1}$, Hanna Lammi ${ }^{1}$, Marketta Niemelä ${ }^{1}$, Kathleen Belhassein ${ }^{2,3}$ \\ Guillaume Sarthou ${ }^{2}$, Antti Tammela ${ }^{1}$, Aurélie Clodic ${ }^{2}$, and Rachid Alami ${ }^{2}$ \\ 1 VTT Technical Research Centre of Finland Ltd \\ firstname. lastname@vtt.fi \\ 2 LAAS-CNRS, Univ. Toulouse, CNRS \\ firstname. lastname@laas.fr \\ 3 CLLE, Univ. Toulouse, CNRS, UT2J
}

\begin{abstract}
Providing guidance to customers in a shopping mall is a suitable task for a social service robot. To be useful for customers, the guidance needs to be intuitive and effective. We conducted a four-phase qualitative study to explore what kind of guidance customers need in a shopping mall, which characteristics make human guidance intuitive and effective there, and what aspects of the guidance should be applied to a social robot. We first interviewed staff working at the information booth of a shopping mall and videotaped demonstrated guidance situations. In a human-human guidance study, ten students conducted seven way-finding tasks each to ask guidance from a human guide. We replicated the study setup to study guidance situations with a social service robot with eight students and four tasks. The robot was controlled using Wizard of $\mathrm{Oz}$ technique. The characteristics that make human guidance intuitive and effective, such as estimation of the distance to the destination, appropriate use of landmarks and pointing gestures, appear to have the same impact when a humanoid robot gives the guidance. Based on the results, we identified nine design implications for a social guidance robot in a shopping mall.
\end{abstract}

Keywords: Shopping mall robot $\cdot$ Robot guidance $\cdot$ Design implications - Multi-phased study · Social robots

\section{Introduction}

Providing information and guidance is a suitable task to a social service robot in a public place such as a shopping mall [19]. Various robots have been tested in offering guidance to mall or store visitors either by route explanation and pointing, or escorting the customer to the target location [4], [7], [9], [10], [20], [5]. Guidance is one of the most desired task for a robot among shopping mall customers and even mall managers, who are aware of the customers challenges in way-finding in their malls [14], [19].

As a guidance facility, compared to mall maps, a robot has some clear advantages. It is a physical device able to move in the same space as humans, and 
humanoid robots have bodily characteristics that are intuitive to understand by humans: "A robot can naturally explain a route by pointing like a human, looking in the same direction as the person is looking, and using such reference terms as 'this way."' [10].

Robot guidance as a form of social interaction between a robot and a human has not been studied much. This is related to perceived usefulness and usability of the robot but also to how the robot should behave in its role as a guide. For instance, in our earlier workshop with shopping mall customers one finding was that a mall robot should not behave as if it was in the position of a human. The robot should not refer to itself as human, for instance, saying "in my view" or "personally I think" [14]. But to what extent a robot guide should mimic a human guide, and how do people perceive a robot as a guide?

In this paper, we report a four-phase study of human guidance and robot guidance. The aim of the study was to understand how a humanoid robot should give guidance in an intuitive and effective way in a shopping mall. We studied this by first gaining understanding of the effective ways to give guidance by human guides and applied the results to guidance behavior of a humanoid robot. Our study questions are:

1. What characteristics of human guidance should be applied to a humanoid robot?

2. What are the design implications for guidance behavior for a social robot?

The study was part of the Multi-Modal Mall Entertainment Robot (MuMMER) project, which develops a socially interactive shopping mall robot with Softbank Robotics Pepper as the robot platform [8]. The robot is intended to provide customer service that complements and extends the current info booth service (i.e. guidance, information and entertainment service). The projects main study site is a large shopping mall Ideapark approximately $30 \mathrm{~km}$ from one of the largest cities in Finland. The mall consists of approx. 200 shops and stores of mainly fashion and leisure equipment. The customers are of all ages from families to older adults. In 2018, there were more than 19000 visitors per day in the mall in average ${ }^{1}$.

\section{Related work}

In the literature, providing guidance is defined as a particular kind of spatial description called "route description" or "route directions". [11] defined it as a set of routes segment, each connecting two important points and explained in a chronological way. The way in which humans communicate spatial knowledge through the route description has been extensively studied both verbally and textually. This has allowed identifying invariants as well as good practices to ensure the success of the task in both urban and interior environments. Through five

\footnotetext{
${ }^{1}$ https://www.kauppakeskusyhdistys.fi/media/kauppakeskusjulkaisu/2018kauppakeskusjulkaisu.pdf (in Finnish).
} 
experiments, Allen [1] identified three basic practices as important for communicating knowledge about routes. They can be summarized as follows: a) respect the spatiotemporal order, b) concentrate on the information about the points of choice and c) use landmarks that the listener can easily identify. This use of reference marks has been called critical information by Tversky in [22] after highlighting that $91 \%$ of the guidance contains additional information (landmarks) to the only actions of reorientation and directions [21], which confirms the results of [6]. Using the terms of [12], guides usually used landmarks when the target places were no longer in the Vista place (being within sight) but in the Environmental space (being reachable through locomotion). In accordance with [22], using landmarks typically occurs when the explained action is a change of direction. In addition, the importance of landmarks and their choice based on salient features during a route description is described in [16].

The choice of the route to explain may have an impact on its understanding and memorization: Morales in [13] argued that the choice is based more on its complexity (the number of stages that compose it) than on its length. The types and frequency of gestures used during route descriptions have been studied in [2]: pointing gestures have been found more frequent than other types of gestures, and their stages to be successful have been highlighted by [24] in the context of collaborative virtual environments.

Many robot guides have been developed based on guidance tasks in a shopping mall. [10] focused on aspects of communication, including user recognition and dialogue history. [17] used a "skeletal" description containing minimal information sets and chose to use only deictic gestures because of their clarity. [19] were interested in the types of questions asked from a robot guide and in the data needed to answer these questions. They also focused on engagement and the reasons why interaction between a human and a robot guide might not start.

\section{Methods and participants}

The research consisted of four parts carried out during the research project (2016-2018, Fig.1): a preliminary interview, a pilot study on human guidance giving, a human-human guidance study and a robot-human guidance study.

First, the preliminary one-hour interview was conducted with a person working at the information booth of the shopping mall to understand what kind of guidance related questions customers ask and how the information workers give guidance. We arranged the interview at the information booth and focused on typical questions from customers and challenging places to guide. We also observed two real situations of guidance giving during the interview. Based on this data, we generated and carried out five example guidance-giving situations and video recorded them.

Second, we carried out a pilot study on human guidance giving in a future location of the mall robot [3]. Based on the preliminary interview, we created a list of 15 locations and items to buy that customers typically ask for receiving guidance and which are located in different parts of the mall. Two researchers 


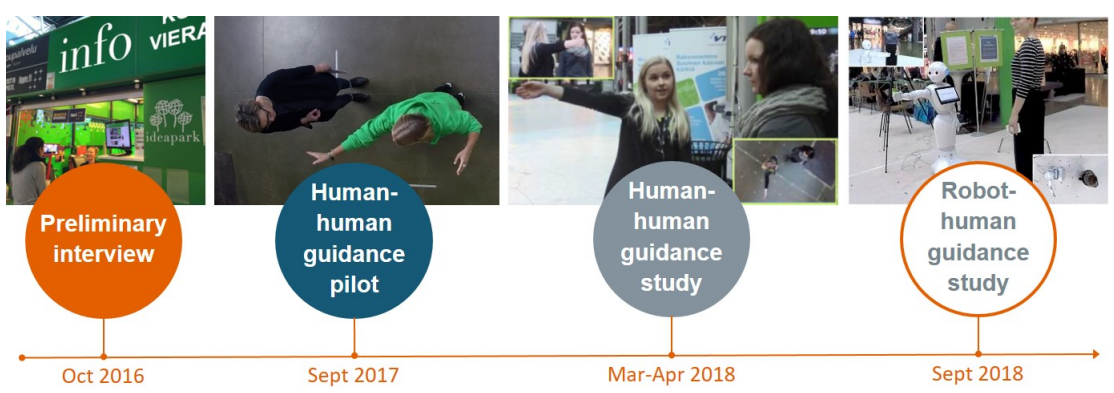

Fig. 1. The study was carried out in four phases during two years.

asked the questions of two persons working at the information booth, who were instructed to give guidance as they would normally do. The situations were video recorded and the guides were briefly interviewed after the sessions.

Third, the human-human guidance study was conducted with 10 volunteer student participants who were not very familiar with the venue. Each participant completed seven way-finding tasks based on the preliminary interviews. The tasks included only locations (e.g. a pharmacy) or a product to buy (e.g. a suitcase), and the participants asked them with their own words. They were also encouraged to ask clarification or further guidance if needed. The tasks included:

1. an easy warm-up task

2. a complex location to guide

3. a product that can be found in several shops

4. two locations in the same question

5. two persons asking guidance to find a place to have lunch together

6. one participant asking guidance and another one interrupting

7. any location or item that participants wanted to ask (preferably according to their real needs if any).

After asking guidance, the participants went to the actual locations and took a photo of the location. We interviewed the participants briefly between the tasks and checked the photos to validate whether they had found the right place. In addition, they all participated in a short individual interview at the end of the study session. After the session, we had a group discussion and revealed them the purpose of the study. They had an opportunity to ask further questions and share their feelings about having a social robot as a guide in the mall. The same person working at the information booth of the shopping mall acted as a guide in all of the sessions. She was asked to give guidance the same way as in typical customer service situation. The sessions were audio recorded and videotaped.

Fourth, the robot-human guidance study replicated the study setting of a human-human guidance study in a slightly smaller scale with 8 volunteer students and 4 guidance tasks for each participant. The tasks were similar to the first four tasks of the human-human study. The findings of the earlier study 
were used in designing the guidance behavior (dialogues and gestures) for the robot. In addition, as part of the dialogue, we tested how participants reacted to personal greetings, e.g. "How has your morning been?". We chose Wizard of $\mathrm{Oz}(\mathrm{WOz})$ technique to control the robot, as an autonomous version that is developed in the research project was not yet stable enough to be used. The wizard was an experienced researcher who stood two meters away from the robot partly behind a pillar and posters covering the computer, not to be too visible to the participants. He controlled the robot to turn, point into appropriate directions and respond with ready-made parts of a dialogue with shortcuts in a WOz interface. In addition, he could write short free-form sentences for the robot to say aloud. Similar to the human-human study, the participants went to the actual locations and took a photo of the location. They were interviewed briefly between the tasks and as a group in the end of the study session. The WOz set-up was revealed to them only after the study. The WOz simulation was found convincing by the participants as none of them had realized the human wizard.

The student participants were recruited through university student email lists and they represented different fields of studies. Most of them had visited the shopping mall a couple of times. Participants who had visited the shopping mall more than once a month were not included. In the human-human study, $6 / 10$ of the participants were female with the mean age of 29 years, ranging from 21 years to 41 years. In the robot-human study $5 / 8$ of the participants were female with the mean age of 34 years, ranging from 24 years to 61 years. The four persons working at the information booth who participated in the first three phases of the study were female and had worked in the mall from a few months to six years.

The study was carried out in compliance with the EU General Data Protection Regulation (GDPR). All participants filled in an informed consent form before participation. The participants were reimbursed with two movie tickets.

The data of the parts of the study were analyzed qualitatively. In this paper, we focus mainly on the data of the interviews. Based on the analysis, we propose design implications for guidance behavior of a social robot.

\section{Results}

\subsection{Effective human guidance}

According to preliminary interviews, typical guidance related questions include specific shops, items to buy, type of the shops (e.g. shoe shops) and recommendations, especially for restaurants. The staff giving guidance highlighted that equality between shops is very important for them, and thus they aim at telling the customer all the options (e.g. all four shoe shops and shops including shoes) or asking further questions of the needs or preferences of the customer (e.g. what kind of food would you like to have for lunch). They do not give direct recommendations, even though they had personal favorites. In addition, they never say that they do not know the response, but always try to help the customer. 
The human-human guidance study revealed means to give guidance in an intuitive and effective way. Even though we designed the guidance tasks to be challenging locations, the participants found all locations according to the guidance given to them. Only one task was such that one of the participants did not find the place easily and needed to use the information board of the shopping center to get further guidance to the location. He was confused because the guide told him the instructions without emphasizing that the location was very close.

Even though the participants found the places relatively easily, the interviews revealed aspects that helped them recalling the instructions or feeling sure that they are on the right route to the location. This was important especially in cases when the location was far away. In general, the participants appreciated short instructions with clear structure accompanied with gestures for pointing the main directions. When the guide gave an estimation of the distance to the location, such as it locating very near or in the very end of the corridor, the participants felt themselves more confident in finding the place. In the cases when they had received instructions to two locations at once, the pointed directions and rough estimations of the distance helped them to recall the instructions to the second location. The instructions were easier to recall, if the guide had used an expression that connected the places together, such as the other destination being located in the totally opposite direction than the first one.

For the places not in sight, the guides typically used landmarks to make the destination and the turnings needed easier to locate. For example, a particular cafe was regularly used as a landmark because it was located at a crossing of two aisles. These results confirm what was highlighted by [21]. The most effective landmarks for the participants were familiar brands having clear signs, confirming the salient features as criteria presented by [16]. One or two landmarks in one guidance dialogue seemed to be a maximum number to be easily recalled.

\subsection{Characteristics of human guidance applied to a social robot}

Based on the results related to effective human guidance, we designed the guidance behavior of the robot to include characteristics that helped the participants to recall the guidance instructions and find the places easily. To make the guidance feel intuitive, we followed the typical structure of giving guidance that the guiding personnel used. One of the guides had a pattern to repeat the location that was asked by the customer first, and we adopted the same confirmation for our robot. In case of a robot, it is crucial that the customer knows that the robot has recognized the destination asked. After that, the robot was designed to give a general remark of the destination first (e.g. it is very close). We regarded this as important for the robot, to give a customer an overall image of the destination first and thus a possibility to orientate to the exact directions.

In the robot-human study, the same characteristics that helped the customers when they were interacting with human guide were found effective also with a robot guide. The participants found the pointing gestures particularly helpful. Similar to the interaction with a human guide, they valued short and structured 
instructions, estimation of the distance to the destination, and appropriate use of landmarks.

The differences to a human guide were not found in the guidance itself, but in the small talk before that. All the participants liked the polite and friendly greetings and way of talking of the robot. They commented that they liked the way the robot looked at them, which made them feel that it is focusing on them and ready to help. However, some felt awkward if the robot asked a personal question in the start of the interaction, such as "How has your morning been?" One participant commented that it feels stupid to tell about your day to a robot, because a robot cannot be truly interested in that.

In general, the participants felt communication with the robot rather natural. The robot initiating the interaction made the situation feel natural and the robot repeating the asked destination confirmed that the robot has understood the questions correctly. The only problems were related to the WOz setup. As the researcher controlling the robot needed to simultaneously manage several aspects of the communication (such as the parts of each guidance dialogue and pointing of directions), the communication was not perfectly smooth and unintentional breaks occurred during the guidance. This made it more difficult for participants to follow the instructions and also know when the interaction ended.

Human-like gestures and way of communication was liked, but a couple of participants commented that besides giving guidance like a human, the robot could use its robotic features and abilities. Unlike a human, a robot could give for example an exact distance to the destination or it could use the tablet in complementing its speech. Naturally, exact distances or tablet use could also confuse users and should be tested before adoption.

\subsection{Design implications for guidance behavior of a social robot}

Based on the results of our multi-phased study, we propose nine implications for designing the guidance behavior of a social robot.

1. Help to start the interaction: Use speech and gaze to receive the attention of the user. Start interaction by friendly greeting and offering help.

2. Confirm the asked location: Before giving guidance, ensure that the request is understood.

3. Give short instructions: Short instructions are easier to understand and recall.

4. Have a clear structure of the instructions with natural rhythm of speech: Clearly structured instructions without long breaks or too fast proceeding help following the instructions and recalling the main points later.

5. Inform distance to the asked location: Give an overall image of the location with an estimation whether it is close or further away.

6. Use gestures (pointing at directions): Helps both initial understanding and recalling the instructions later.

7. Use landmarks when appropriate: Salient landmarks as well as ones located in the crossings of aisles are helpful. However, include only 1-2 landmarks in one guidance case. 
8. Ensure equality between retails: Instead of personal recommendation, try finding out the preferences of the customer.

9. Stay flexible: Give the customer a possibility to ask for repeating an instruction or to interrupt the situation. When the response includes several options, the customer should be able to ask further questions (e.g. which is the closest option). If the robot cannot answer a question, it could advice the customer to continue the case with the personnel of the mall.

\section{Discussion}

The aim of our research was to find out how we should design a social service robot to give guidance in an intuitive and effective way in a shopping mall. Based on our multi-phased study, we identified design implications and explored whether a humanoid robot should guide like a human.

According to our results, the participants felt human-like guidance behavior intuitive and the characteristics used by a human guide were effective in robothuman interaction. Our findings are in line with the principles introduced by Allen [1], emphasizing the clear structure of guidance as well as a chose of landmarks so that the customer can find the points of interest quickly and easily. Thus, the requirements and expectations of a route description by a robot do not seem to differ from a human-human guidance. However, asking additional personal questions ("How your morning has been?") was felt suitable for a human only. It seems that even though the structure of the communication and the aspects of making the guidance easy to understand and recall, seem to be applicable to humanoid robots, the robot should not pretend to be a human or replicate the human behavior besides functional aspects of it. This supports our earlier findings that a mall robot should not behave as if it were in the position of a human [14]. However, the result might be different in a study that would focus on e.g. free communication with a robot instead of functional task, such as guidance.

The limitations of this study include a small sample size and practical problems with the WOz setup. External validity is high as the guidance studies were carried out in the field, in an actual shopping mall with realistic way-finding tasks. However, the results cannot be directly generalized to all customer groups or other shopping malls. The participants were students, who do not make a representative sample of the mall customer population. For instance, student participants might have a more positive attitude towards a robot guide than the mall customers in average, which would make them willing to listen to the robots advice more carefully than an average customer would. In some earlier human-robot interaction studies in the MuMMER project, we have recruited participants in the field [15]. However, this was not possible for this study due to the study setting, which required the participants to commit for 2-3 hours to the study. Therefore, we have to be cautious when drawing conclusions from the results. Furthermore, we assume the attitudes to the human-like behavior of robots to be strongly dependent on the cultural context, and thus it would be interesting to extend the study also to other cultures. 
Our results have been used in developing software components for the robot that will be deployed in the shopping mall in the end of the research project. The components include software for route search and the guidance explanations generation [18] and planning of the positioning of the human and the robot so that they have a common perspective while pointing [23]. During the deployment, we will extend our research to more realistic guidance situations with the autonomous robot.

\section{Conclusions}

In this paper, we presented a four-phased qualitative study to explore which means make human guidance intuitive and effective, and whether the means have the same impact in guidance given by a humanoid service robot. We proposed nine design implications and discussed whether a robot should guide like a human. Our results give practical insights to human-robot interaction designers and contribute to the literature of robot guidance as a form of social interaction.

\section{Acknowledgements}

We thank Olivier Canévet for creating a Wizard of Oz user interface which we used in this study and Petri Tikka for giving technical support. We also thank Ideapark for their cooperation and the volunteer participants of the study. This work has been supported by the European Union's Horizon 2020 research and innovation program under grant agreement No. 688147 (MuMMER project).

\section{References}

1. Allen, G.L.: Principles and practices for communicating route knowledge. Applied Cognitive Psychology 14(4), 333-359 (2000)

2. Allen, G.L.: Gestures accompanying verbal route directions: do they point to a new avenue for examining spatial representation? Spatial cognition and computation 3(4), 259-268 (2003)

3. Belhassein, K., et al.: Human-Human Guidance Study. tech-report hal-01719730, LAAS-CNRS, CLLE, VTT (2017)

4. Brscic, D., Ikeda, T., Kanda, T.: Do you need help? a robot providing information to people who behave atypically. IEEE Transactions on Robotics 33(2) (2017)

5. Clodic, A., et al.: Rackham: An Interactive Robot-Guide. In: 15th IEEE RO-MAN (2006)

6. Denis, M.: The description of routes: A cognitive approach to the production of spatial discourse. Cahiers de Psychologie Cognitive 16, 409-458 (1997)

7. Díaz, M., Paillacho, D., Angulo, C., Torres, O., Gonzlez, J., Albo-Canals, J.: A week-long study on robot-visitors spatial relationships during guidance in a sciences museum. In: 9th ACM/IEEE International Conference on HRI. pp. 152-153 (2014)

8. Foster, M.E., et al.: The MuMMER Project: Engaging Human-Robot Interaction in Real-World Public Spaces. In: 8th International Conference on Social Robotics, ICSR 2016 (2016) 
9. Gross, H., et al.: TOOMAS: Interactive shopping guide robots in everyday use - final implementation and experiences from long-term field trials. In: 2009 IEEE/RSJ IROS. pp. 2005-2012 (2009)

10. Kanda, T., et al.: A Communication Robot in a Shopping Mall. IEEE Transactions on Robotics 26(5), 897-913 (2010). https://doi.org/10.1109/TRO.2010.2062550

11. Kopp, S., et al.: Trading Spaces: How Humans and Humanoids Use Speech and Gesture to Give Directions. In: Nishida, T. (ed.) Wiley Series in Agent Technology, pp. 133-160. John Wiley \& Sons, Ltd, Chichester, UK (2007)

12. Montello, D.R.: Scale and multiple psychologies of space. In: Frank, A.U., Campari, I. (eds.) Spatial Information Theory A Theoretical Basis for GIS. pp. 312-321. Springer Berlin Heidelberg, Berlin, Heidelberg (1993)

13. Morales, Y., Satake, S., Kanda, T., Hagita, N.: Building a Model of the Environment from a Route Perspective for HumanRobot Interaction. International Journal of Social Robotics 7(2), 165-181 (2015)

14. Niemelä, M., Heikkilä, P., Lammi, H., Oksman, V.: A social robot in a shopping mall: studies on acceptance and stakeholder expectations (in publication). In: O. Korn (Ed.), Social Robots: Technological, Societal and Ethical Aspects of HumanRobot Interaction. Springer. (2019)

15. Niemelä, M., Arvola, A., Aaltonen, I.: Monitoring the acceptance of a social service robot in a shopping mall: First results. In: Proceedings of the Companion of the 2017 ACM/IEEE HRI. ACM (2017)

16. Nothegger, C., Winter, S., Raubal, M.: Selection of Salient Features for Route Directions. Spatial Cognition \& Computation 4(2), 113-136 (2004)

17. Okuno, Y., et al.: Providing route directions: design of robot's utterance, gesture, and timing. In: 4th ACM/IEEE International Conference on HRI (2009)

18. Sarthou, G., Clodic, A., Alami, R.: Semantic spatial representation: a unique representation of an environment based on an ontology for robotic applications. In: Proceedings of the Combined Workshop SpLU-RoboNLP. pp. 50-60. Association for Computational Linguistics (2019)

19. Satake, S., Nakatani, K., Hayashi, K., Kanda, T., Imai, M.: What should we know to develop an information robot? PeerJ Computer Science 1 (2015)

20. Triebel, R., et al.: SPENCER: A Socially Aware Service Robot for Passenger Guidance and Help in Busy Airports. 10th International Conference on Field and Service Robotics, Springer, Toronto, Canada (2015)

21. Tversky, B., Lee, P.U.: How Space Structures Language. In: Freksa, C., Habel, C., Wender, K.F. (eds.) Spatial Cognition: An Interdisciplinary Approach to Representing and Processing Spatial Knowledge. Lecture Notes in Computer Science, Springer (1998)

22. Tversky, B., Lee, P.U.: Pictorial and Verbal Tools for Conveying Routes. In: Freksa, C., Mark, D.M. (eds.) Spatial Information Theory. Cognitive and Computational Foundations of Geographic Information Science. pp. 51-64. Lecture Notes in Computer Science, Springer Berlin Heidelberg (1999)

23. Waldhart, J., Clodic, A., Alami, R.: Planning Human and Robot Placements for Shared Visual Perspective. In: Workshop on Robotic Co-workers 4.0 in IEEE/RSJ International Conference on Intelligent Robots and Systems (IROS) (2018)

24. Wong, N., Gutwin, C.: Where are you pointing? the accuracy of deictic pointing in CVEs. In: Proceedings of the SIGCHI conference on human factors in computing systems. pp. 1029-1038. ACM Press (2010) 